\title{
Microencapsulação por spray drying dos compostos bioativos do extra- to aquoso de erva mate (Ilex paraguariensis) crioconcentrado
}

\author{
Microencapsulation by spray drying of bioactive compounds of yerba mate extract \\ aqueous (Ilex paraguariensis) freeze concentration
}

\author{
Graciele Lorenzoni Nunes ${ }^{1}$, Cristiano Ragagnin de Menezes ${ }^{2}$ \\ ${ }^{1}$ Doutoranda em Ciência e Tecnologia dos Alimentos, Departamento de Tecnologia e Ciência de Alimentos, \\ Universidade Federal de Santa Maria, Santa Maria, Brasil \\ graci_nunnes@hotmail.com
}

${ }^{2}$ Professor Doutor, Departamento de Tecnologia e Ciência de Alimentos, Universidade Federal de Santa Maria, Santa Maria, Brasil cristiano.ufsm@gmail.com

\begin{abstract}
Resumo
Nos diversos trabalhos científicos têm sido demonstrados os efeitos benéficos da Ilex paraguariensis, principalmente relacionados às suas propriedades antioxidantes, devido ao alto teor de compostos fenólicos presentes em seu extrato aquoso. Dessa forma, a aplicação de tecnologias de concentração, tais como, a crioconcentração torna-se relevante, uma vez que promove a concentração de produtos alimentares, através do congelamento e subsequente separação de uma parte da água congelada, a partir de um produto líquido, resultando em um aumento no teor de compostos fenólicos do extrato aquoso de folhas de erva mate, e na minimização de perdas destes compostos. Devido ao alto teor de compostos fenólicos, o extrato crioconcentrado de erva mate se torna instável, sendo susceptível à degradação pela ação de alguns fatores, como presença de oxigênio, luz, pH e temperatura e assim a aplicação de processos de microencapsulação, tais como a atomização se apresenta como uma alternativa importante na melhoria da estabilidade dos compostos fenólicos do extrato aquoso crioconcentrado das folhas de erva mate.
\end{abstract}

Palavras-chave: Erva mate. Crioconcentração. Microencapsulação. Spray dryer.

\begin{abstract}
Several scientific studies have shown the beneficial effects of yerba mate, mainly related to its antioxidant properties, due to the high content of phenolic compounds present in the aqueous extract. In this context, the application concentration technologies, such as the freeze concentration becomes relevant, since it promotes concentration of food by freezing and subsequent separation of a part of the frozen water, from a liquid product, resulting in an increase in the content of phenolic compounds from aqueous extract of yerba mate leaves, and minimizing losses of these compounds. Due to the high content of phenolic compounds, the freeze concentration of yerba mate extract becomes unstable and is susceptible to degradation by the action of some factors such as the presence of oxygen, light, $\mathrm{pH}$ and temperature and so the application of microencapsulation processes such as atomization presents itself as an important alternative in improving the stability of the phenolic compounds of the aqueous extract of freeze concentration of yerba mate leaves.
\end{abstract}

Keywords: Ilex paraguarienses. Freeze concentration. Microencapsulation. Spray drying. 


\section{Introdução}

A erva mate (Ilex paraguariensis) é uma planta que apresenta, em suas folhas, uma diversidade de compostos químicos que são reconhecidos por exercerem efeitos benéficos ao organismo. Os compostos fenólicos estão presentes em grandes quantidades nas folhas de erva mate e a eles são atribuídas às propriedades antioxidantes apresentadas por esta planta (BASTOS et al., 2007; DE MEJIA et al., 2009; BRACESCO et al., 2011; ANESINI et al., 2012).

A crioconcentração é uma tecnologia que promove a concentração de produtos alimentares, através do congelamento e subsequente separação de uma parte da água congelada, a partir de um produto líquido (BELÉN et al., 2012). Este método foi empregado com sucesso por Boaventura et al. (2013) na concentração dos compostos fenólicos do extrato aquoso de folhas de erva mate, resultando em um aumento no teor de compostos fenólicos e na minimização de perdas destes compostos. No entanto, devido ao alto teor de compostos fenólicos, o extrato crioconcentrado de erva mate se torna instável, sendo susceptível à degradação pela ação de diversos fatores, como presença de oxigênio, luz, pH e temperatura (BAKOWSKA; KUCHARSKA; OSZMANSKI, 2003). Processos de microencapsulação, tais como a atomização, se apresenta como uma alternativa na melhoria da estabilidade dos compostos fenólicos presentes no extrato aquoso das folhas de erva mate crioconcentrado. Esta tecnologia consiste no empacotamento de partículas, através da utilização de um agente encapsulante, com o intuito de proteger e prolongar sua funcionalidade, sendo também uma alternativa para modificar as características de um produto e melhorar sua aparência (MORETTI et al., 2003; AZEREDO, 2005; KUANG et al., 2010). Diversos agentes encapsulantes estão disponíveis para este processo, no entanto, para a aplicação em alimentos, é comumente utilizada goma arábica, maltodextrina em diferentes graus de dextrose equivalente (DE) e algumas proteínas (SHAHIDI; HAN, 1993). A maltodextrina possui alta solubilidade em água, baixa viscosidade, além de produzir soluções incolores, sendo um ingrediente útil na indústria de alimentos (GHARSALLAOUI et al., 2007).

Estudos recentes têm sido desenvolvidos com o objetivo de encapsular o extrato de erva mate por meio de diferentes métodos e com distintas finalidades (BERTÉ, 2011; ALEX, LORENA, MIRIAN, 2013; DELADINO, NAVARRO, MARTINO, 2013; LOPÉZ-CORDOBA; DELADINO; MARTINO, 2014; CÓRDOBA et al., 2014).

\subsection{Erva mate}

A erva mate é uma planta arbórea, pertencente à família Aquifoliaceae e ao gênero Ilex, originária da América do Sul e nativa de regiões de clima temperado (CARVALHO, 2003). O seu cultivo é conhecido por silvicultura, ocor- rendo naturalmente no leste do Paraguai, sul do Brasil e noroeste da Argentina, com dimensões de ocupação de aproximadamente $540.000 \mathrm{Km}^{2}$, sendo que o Brasil corresponde a $450.000 \mathrm{~km}^{2}$, representando cerca de $5 \%$ do território nacional (RESENDE et al., 2000; ROTTA; OLIVEIRA, 2005). A maior produção de erva mate, em âmbito mundial é encontrada na Argentina com 701 mil toneladas de erva mate verde, seguida pelo Brasil com 513 mil toneladas de erva mate verde e Paraguai com 94 mil toneladas (BRASIL, 2014). O Brasil é o país com maior área de produção ervateira, no entanto, a Argentina é a maior exportadora mundial de erva mate, e o Uruguai, o país que apresenta o maior consumo per capita (BRACESCO et al., 2011).

O consumo de erva mate acontece principalmente em diferentes tipos de bebidas, tais como o chimarrão e o tererê, que são preparados com as folhas verdes e o chá mate, preparado com as folhas tostadas (BASTOS et al., 2007; BRACESCO et al., 2011). A cultura da erva mate e o hábito de beber chimarrão desempenham importante papel econômico e sociocultural na região sul do Brasil, sendo essa bebida considerada um forte instrumento de socialização do povo sulino, e o cultivo e a industrialização da erva, mate uma importante alternativa econômica à agricultura familiar desses estados (CORREA et al., 2011).

Até pouco tempo o setor ervateiro dependia quase que exclusivamente da comercialização da erva mate na sua forma tradicional de chimarrão, o que limitava o mercado às regiões onde era produzida. Na última década, juntamente com o uso contínuo de bebidas tradicionais à base de erva mate, a Ilex paraguariensis tem sido utilizada no desenvolvimento de cervejas, cremes, doces e outros usos não tradicionais (VIEIRA et al., 2008). Neste contexto, muitos estudos têm sido conduzidos com o intuito de identificar formas comerciais alternativas para a aplicação da erva mate, visando agregar valor a essa importante matéria prima regional (VALDUGA, 2002).

\section{Composição química e propriedades bio- lógicas da erva mate}

A grande diversidade de compostos químicos com propriedades biológicas encontrados nas folhas de erva mate tem sido alvo de diversas pesquisas. Estes estudos têm demonstrado as diferentes propriedades apresentadas pela erva mate, tais como, atividade antioxidante (BOAVENTURA et al., 2012; FERNANDES et al., 2012), efeito protetor contra danos induzidos ao DNA (MIRANDA et al., 2008), atividade de vasodilatação (PAGANINI STEIN et al., 2005), efeitos sobre a inibição da glicação e aterosclerose (MOSIMANN; WILHELM-FILHO; DA SILVA, 2006), efeitos termogênicos (POMILIO et al., 2002; ARCARI et al., 2009), efeito na melhoria da tolerância à glicose (KANG et al., 2012; PEREIRA et al., 2012) e efeitos 
anti-inflamatórios (ARCARI et al., 2009; PIMENTEL et al., 2012). Estas propriedades estão associadas aos seus constituintes químicos, dentre eles, saponinas, metilxantinas, minerais e vitaminas e, especialmente, ao teor de compostos fenólicos (BRACESCO et al., 2003; HECK; DE MEJIA, 2007; MATSUMOTO, 2009; FILIP et al., 2010).

As metilxantinas são constituintes químicos derivados de bases púricas, as quais possuem nitrogênio heterocíclico e apresentam atividade fisiológica marcante (MEINHART et al., 2010; SILVA et al., 2011). Na erva mate, a principal metilxantina encontrada é a cafeína, seguida pela teobromina e em menores quantidades teofilina (CLIFFORD; RAMIREZ-MARTINEZ, 1990). Essa classe de compostos é responsável por exercer efeitos estimulantes ao sistema nervoso central, cardiovascular, renal, digestivo e por elevar a utilização de gordura como fonte energética (DERMARDEROSIAN, 2001; WICHTL, 2003; RATES, 2007, SILVA et al., 2011).

As saponinas são substâncias quimicamente constituídas por uma porção hidrofóbica triterpênica ou esteróide, ligada a uma ou mais cadeias de açúcares, em geral, glicose, galactose, ácido glicurônico, xilose ou metilpentose (FRANCIS et al., 2002). As saponinas são altamente solúveis em água, propriedade que confere às bebidas à base de erva mate o gosto amargo e a formação de espuma (SPARG et al., 2004). Na erva mate, a porção aglicona das saponinas é comumente composta por dois triterpenóides, os ácidos ursólico e oleanólico (GNOATTO et al., 2008).

As propriedades químicas das saponinas incluem, principalmente, sua habilidade como agente espumante, sendo explorada pela indústria como aditivos para alimentos e cosméticos. Também detêm potencial para outras aplicações industriais como conservantes e modificadores de sabor (SAN MARTÍN; BRIONES, 1999; GUCLU-USTUNDAG; MAZZA, 2007). Com relação à atividade biológica, as saponinas são reconhecidas por conterem propriedades de complexação com esteroides, proteínas e fosfolipídios, além de propriedades de formação de poros em membranas celulares, destacando-se a atividade hemolítica, antiviral e hipocolesterolêmica (FRANCIS, et al., 2002; SCHENKEL et al., 2003). As saponinas isoladas da erva mate, bem como do extrato aquoso da planta, inibiram in vitro a difusão passiva de ácido cólico, mimetizando a inibição da absorção de ácidos biliares pelas células intestinais (FERREIRA et al., 1997), a qual tem efeito na diminuição do colesterol. Em um estudo realizado por De Morais et al. (2009) foi observada uma redução significativa do colesterol após 40 dias de consumo de infusão de erva mate por indivíduos hipercolesterolêmicos, a qual pode estar relacionada principalmente à atividade das saponinas presentes na planta.

Os compostos fenólicos são substâncias que contêm, em sua estrutura, um anel aromático em que, ao menos um hidrogênio é substituído por um grupamento hidroxila (SIMÕES et al., 2000; HECK; DE MEJIA, 2007;
LEOPOLDINI et al., 2011). Na erva mate estão presentes, principalmente, na forma de ácidos fenólicos, o ácido cafeico, o ácido clorogênico e seus isômeros e, em menor proporção, na forma de flavonoides (rutina, quercetina e canferol) (BRAVO; GOYA; LECUMBERRI, 2007).

Os compostos fenólicos apresentam grande importância na defesa a radicais livres produzidos, normalmente, pelo metabolismo das células ou em resposta a fatores externos, destacando-se sua ação antioxidante (SOTO-VACA, 2012).

\section{Atividade antioxidante da erva mate}

Dentre as atividades biológicas proporcionadas pela erva mate, uma das principais e mais conhecidas é a atividade antioxidante, a qual possui a propriedade de combater os radicais livres e as espécies reativas de oxigênio. Na erva mate a atividade antioxidante é fortemente relacionada ao teor de compostos fenólicos presentes em suas folhas (FANG; YANG; WU, 2002; HECK; SCHMALKO; GONZALEZ DE MEJIA, 2008). Há relatos em estudos sobre o elevado potencial antioxidante do extrato aquoso de erva mate in vitro. Filip et al. (2000) analisaram a atividade antioxidante de plantas do gênero Ilex, observando que a erva mate (Ilex paraguariensis) apresentou maior atividade e que essa propriedade era preservada na bebida. Mejia et al. (2010) mostraram que a erva mate apresentou eficiente inibição in vitro de câncer de cólon, além disso, a atividade antioxidante da erva mate também foi relacionada com a inibição da oxidação da lipoproteína de baixa densidade (LDL) (GUGLIUCCI; STAHL, 1995; DA SILVA et al., 2008; BOAVENTURA et al., 2012).

Em alimentos, os danos oxidativos podem afetar macro e micronutrientes presentes nos produtos alimentícios. Neste contexto, estudos vêm sendo desenvolvidos com o interesse de encontrar ingredientes ou compostos naturais que tenham propriedades antioxidantes. Uma pesquisa realizada por Campos et al. (2007) demonstrou que a atividade antioxidante apresentada pelo extrato de erva mate, quando adicionado ao salame, foi capaz de inibir a oxidação lipídica.

Para avaliar a atividade antioxidante in vitro, diversas técnicas têm sido utilizadas, entre elas, os métodos baseados nas reações de transferência de elétron (TE), destacando-se os métodos DPPH (2,2- difenil-1-picril-hidrazila), ABTS [2,2, azinobis (3-etilbenzotiazolina-6-ácido sulfônico)] e FRAP (Poder Antioxidante de Redução do Ferro) (DUDONNÉ et al., 2009) e, também, o método de ORAC (Capacidade de Absorção de Radicais Oxigênio), que é baseado em reações de transferências de átomos de hidrogênio (HUANG; OU; PRIOR, 2005).

Dentre estes métodos, o DPPH é uma técnica de sequestro de radicais livres, que consiste na descoloração de uma solução composta por radicais estáveis DPPH•, de cor violeta quando ocorre a adição de substâncias an- 
tioxidantes (BRAND-WILLIAMS; CUVELIER; BERSET, 1995; HUANG; OU; PRIOR, 2005), ou seja, seu princípio é baseado na transferência de elétrons de um composto antioxidante para um oxidante. Nesta metodologia, têmse, como vantagens, a simplicidade e a capacidade de avaliar a atividade antioxidante de produtos naturais em condições de temperatura ambiente (MOLYNEUX, 2004; PRIOR; WU; SCHAICH, 2005; ROGINSKY; LISSI, 2005).

O método FRAP baseia-se na capacidade de redução do complexo férrico-tripiridiltriazina ( $\left.\mathrm{Fe}^{3+}-\mathrm{TPTZ}\right)$ a complexo ferroso ( $\mathrm{Fe}^{2+}$-TPTZ) na presença de um antioxidante e em condições ácidas. O complexo formado por esta reação possui uma coloração azul intensa, o que leva ao aumento da absorbância (BENZIE; STRAIN, 1996; PÉREZ-JIMÉNEZ; SAURA-CALIXTO, 2006).

\section{Concentração de compostos bioativos}

A concentração de compostos bioativos tem por objetivo eliminar parcialmente o líquido extrator, levando à obtenção de um produto concentrado, com viscosidade e consistência variáveis (SONAGLIO et al., 2007).

O conteúdo de água presente nos alimentos é comumente removido, a fim de reduzir custos de armazenamento e diminuir a atividade de água para garantir a estabilidade microbiológica do produto. Os processos tecnológicos aplicados para este fim incluem processos de concentração, que são aqueles que aumentam o conteúdo de sólidos em torno de $60 \%$ e desidratação, em que se reduz o teor de água a menos de 10 \% (SILVA, 2003).

Na indústria de alimentos, a concentração por evaporação é um processo rotineiramente utilizado por se tratar de um sistema, relativamente, simples e por apresentar custo de operação limitado, na medida em que podem ser aplicados múltiplos efeitos ou se utilizar bombas de calor econômicas (AIDER; DE HALLEUX; AKBACHE, 2007). No entanto, este processo pode causar efeitos nas qualidades sensoriais e nutricionais dos produtos, uma vez que temperaturas acima de $60^{\circ} \mathrm{C}$ podem provocar perdas importantes de compostos voláteis e aromáticos, vitaminas, proteínas e compostos termoinstáveis (FRYER; ROBBINS, 2005; LEWICK, 2006; NAKAGAWA; MAEBASHI; MAEDA, 2010).

A tecnologia de concentração por membranas tem sido utilizada com sucesso como método alternativo para concentrar compostos fenólicos (XU; WANG, 2005). Neste processo são utilizadas baixas temperaturas e mínimo consumo energético, mantendo a qualidade dos compostos termoinstáveis. Murakami et al. (2011) e Prudêncio et al. (2012) aplicaram a tecnologia de nanofiltração para concentrar os compostos fenólicos das folhas e talos da erva mate, respectivamente. Por ambos os estudos foi verificado um aumento significativo do teor de compostos fenólicos totais do extrato aquoso de folhas e talos de erva mate após a aplicação desta tecnologia de concentração. No entanto, a concentração por membranas apresenta limitações, devido a alguns fenômenos decorrentes do processo, como o efeito fouling (entupimento dos poros da membrana) (TURAN; ATES; INANC, 2002; AIDER; DE HALLEUX; AKBACHE, 2007). A crioconcentração, tecnologia recentemente aplicada por Boaventura et al. (2013) para concentrar os compostos fenólicos do extrato aquoso de erva mate, vem sendo reconhecida como tecnologia promissora na concentração de bioingredientes em produtos alimentares líquidos (AIDER; DE HALLEUX, 2009).

A crioconcentração é uma tecnologia aplicada com o objetivo de promover a concentração de produtos alimentares, através do congelamento e da subsequente separação de uma parte da água congelada, a partir de um produto líquido (BELÉN et al., 2012). Este processo vem sendo reconhecido como uma técnica de concentração promissora a ser utilizada para evitar a perda da qualidade de produtos líquidos, tais como extratos de plantas (CHABAROV; AIDER, 2014). Dessa forma, é uma alternativa às técnicas convencionais utilizadas na concentração de produtos alimentares, como a evaporação e a tecnologia de membranas (SILVA, 2003).

Há diferentes métodos para a aplicação da tecnologia de crioconcentração, tais como o método de cristalização por suspensão, que é a forma de crioconcentração com sistema mais complexo. Este sistema envolve trocadores de calor de paredes raspadas para a geração dos cristais de gelo; tubo de recristalização para o crescimento dos cristais e torre de lavagem para a separação dos cristais de gelo. Este método apresenta alto custo de execução, limitando assim sua aplicação à indústria de alimentos (MIYAWAKI et al., 2005; AIDER; DE HALLEUX, 2009).

A crioconcentração progressiva é um método no qual ocorre a formação de apenas um único grande cristal de gelo, facilitando a separação entre o cristal de gelo e a solução mãe. Esse sistema é composto de um compartimento cilíndrico, um banho de resfriamento e um sistema de condução da amostra, sendo que o cristal é formado na superfície de resfriamento do reservatório de cristalização (MIYAWAKI et al., 2005; AIDER; DE HALLEUX, 2009). A crioconcentração eutética é baseada no princípio da separação de sais inorgânicos e suas soluções aquosas, onde ocorre simultaneamente a cristalização dos sais e do gelo. Este processo é realizado pelo resfriamento da solução perto ou abaixo da temperatura eutética. O ponto eutético é onde todos os constituintes cristalizam, simultaneamente, à temperatura de fusão da solução líquida (HIMAWAN et al., 2002; AIDER; DE HALLEUX, 2009). A separação entre os cristais de sal e o gelo é realizada gravitacionalmente, tendo em conta as diferenças na densidade dos dois sólidos formados (AIDER; DE HALLEUX, 2009).

A crioconcentração em blocos é um método que consiste no congelamento da solução alimentar líquida, podendo ocorrer de forma parcial ou total. No congelamento parcial, ocorre a introdução de um tubo refrigerante no centro da solução de alimentação. Na crioconcentração 
em blocos total, ocorre o congelamento total de uma solução, seguido por um procedimento de descongelamento parcial, sob separação gravitacional simples (AIDER; DE HALLEUX; AKBACHE, 2007; AIDER; DE HALLEUX, 2009). Assim, este método consiste de três etapas: congelamento, descongelamento e a separação do líquido concentrado (MORENO et al., 2014).

A crioconcentração em blocos vem sendo relatada como promissora e eficaz à obtenção de produtos alimentícios com maior valor nutritivo e com maior preservação das propriedades sensoriais (AIDER; DE HELLEUX; AKBACHE, 2007; AIDER; DE HALLEUX, 2008; AIDER; DE HALLEUX; MENILKOVA, 2009; MORENO et al., 2014).

Na indústria alimentícia, a crioconcentração tem sido empregada na concentração de sucos cítricos, soluções diluídas e extratos de chá e café, como também na concentração de soro de queijos (ANON, 1993; SÁNCHEZ et al., 2010; SÁNCHEZ et al., 2011; MORENO et al., 2014). Boaventura et al. (2013) aplicaram a crioconcentração em blocos total no extrato aquoso de folhas de erva mate, e verificaram que esta tecnologia foi eficiente na concentração e preservação da qualidade nutricional dos compostos fenólicos, apresentando quantidades crescentes destes compostos em todos os estágios de concentração.

No entanto, o extrato crioconcentrado das folhas de erva mate se torna altamente instável pela presença de altos teores de compostos fenólicos, uma vez que esses compostos são susceptíveis à degradação pela ação de diversos fatores, como a presença de oxigênio, luz, $\mathrm{pH}$ e temperatura (BAKOWSKA et al., 2003). Neste contexto, a aplicação de processos de microencapsulação é uma alternativa para aumentar a estabilidade dos compostos fenólicos presentes no extrato aquoso de folhas de erva mate crioconcentrado.

\section{Microencapsulação}

A utilização de técnicas para o processo de encapsulação de polifenóis, tais como secagem por atomização, coacervação, complexação, co-cristalização, nanoencapsulação, liofilização e emulsão têm recebido grande destaque (FANG; BHANDARI, 2010; MUNIN; EDWARDS-LÉVY, 2011). Recentemente, extratos de erva mate foram encapsulados através das técnicas de co-cristalização, liofilização e gelificação iônica (DELADINO; NAVARRO; MARTINO, 2013; LÓPEZ-CORDOBA et al., 2014; LÓPEZ-CORDOBA; DELADINO; MARTINO, 2014).

A técnica de microencapsulação já é conhecida há muitas décadas, no entanto vem ganhando espaço em aplicações nas mais diversas áreas, tais como a farmacêutica, alimentar e cosmética (SUAVE et al., 2006). Seu conceito tem como base a idealização do modelo celular, no qual o núcleo é envolvido por uma membrana semipermeável que o protege do meio externo e, ao mesmo tempo, controla a entrada e saída de substâncias na célula (RÉ, 2006). Assim, a microencapsulação compreende um processo em que ocorre a incorporação de substâncias de interesse (núcleo ou material ativo) em um sistema de revestimento (material de parede, carreador ou agente encapsulante), obtendo-se microcápsulas com um diâmetro que varia entre 1 e $1000 \mu \mathrm{m}$ (MADENE et al., 2006; OBEIDAT, 2009; TIWARI, et al., 2010). As substâncias que serão encapsuladas se apresentam, geralmente, no estado líquido ou sólido, podendo também ser um gás (TRINDADE et al., 2008).

Na indústria de alimentos a microencapsulação é utilizada com o objetivo de revestir um ou mais ingredientes ou aditivos por um agente encapsulante de natureza comestível, modificando e melhorando a aparência e as propriedades de algumas substâncias. Outras finalidades deste processo podem ser descritas, como a diminuição das interações da substância encapsulada em relação aos fatores ambientais, impedindo perdas sensoriais e nutricionais; mascarar substâncias com sabores indesejáveis; melhorar a solubilidade da substância encapsulada e a sua incorporação em sistemas secos; permitir que a liberação da substância encapsulada seja modificada, ocorrendo de forma lenta ou a partir de determinado estímulo; reduzir a velocidade de evaporação de substâncias voláteis e aumentar o tempo de armazenamento das substâncias a encapsular (AZEREDO, 2005; KUANG et al., 2010).

Diversos métodos (químicos, físicos, ou físico-químicos) podem ser utilizados na realização do processo de microencapsulação (Tabela 1). A diferença entre eles está no envolvimento ou aprisionamento do material a encapsular pelo agente encapsulante. Dessa forma, devem ser consideradas as propriedades físico-químicas do material a encapsular e do agente encapsulante, a aplicação e finalidade, o tamanho, textura e forma das microcápsulas, bem como o mecanismo de liberação do material a ser encapsulado (BANSODE et al., 2010). Deste modo, o método de microencapsulação deve ser simples, reprodutível, rápido e fácil de transportar a escala industrial (SILVA et al., 2003).

Entre os métodos existentes, o de spray drying é um dos mais antigos, tendo sido originalmente utilizado na década de 30, para preparar os primeiros compostos contendo sabores encapsulados (WILSON; SHAH, 2007). O procedimento para a realização deste método compreende algumas etapas (Figura 1): primeiramente, a substância a ser encapsulada é homogeneamente dispersa ou dissolvida em uma solução aquosa ou dispersão, contendo o agente encapsulante. Depois, o sistema é atomizado em uma corrente de ar quente que vai promover a evaporação do solvente, obtendo-se a rápida solidificação das gotículas que depois serão recolhidas no ciclone. As variáveis deste processo envolvem a temperatura de entrada e saída de ar do sistema, o fluxo de ar ou fluído de arraste, a distribuição da temperatura e umidade, o tempo de permanência e temperatura da 
câmara. Estes parâmetros determinarão a eficiência do processo, juntamente, com as características do agente encapsulante (tamanho de moléculas, solubilidade) e características do material ativo (polaridade, pressão de vapor, tamanho de molécula) (RÉ, 1998; KISSEL et al., 2006).

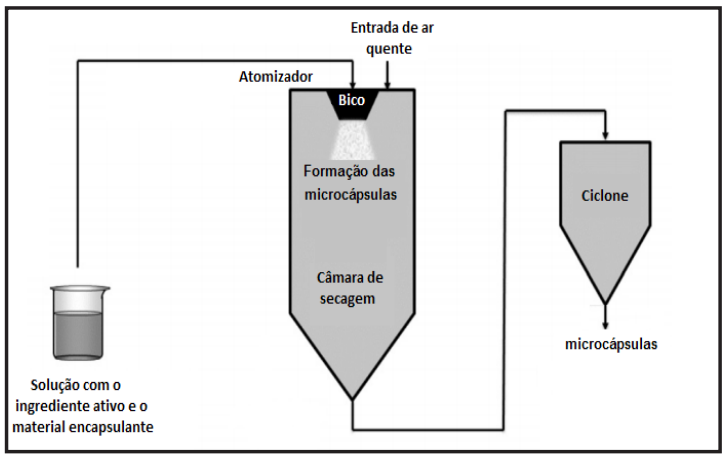

Figura 1 - Representação esquemática da microencapsulação por spray drying

Fonte: adaptado de Burgain et al. (2011)

As microcápsulas fisicamente são caracterizadas pela sua forma esférica e por apresentar aspecto sólido. Nos métodos de microencapsulação, diferentes grupos morfológicos podem ser observados, sendo dois os mais comumente visualizados: (1)

mononucleares, onde o material ativo fica concentrado próximo ao centro, rodeado pelo agente encapsulante ou, (2) multinucleares, onde o agente encapsulante forma uma rede tridimensional com vários materiais ativos que podem estar adsorvidos, incorporados ou ligados covalentemente à matriz (Figura 2) (FANG; BHANDARI, 2010).
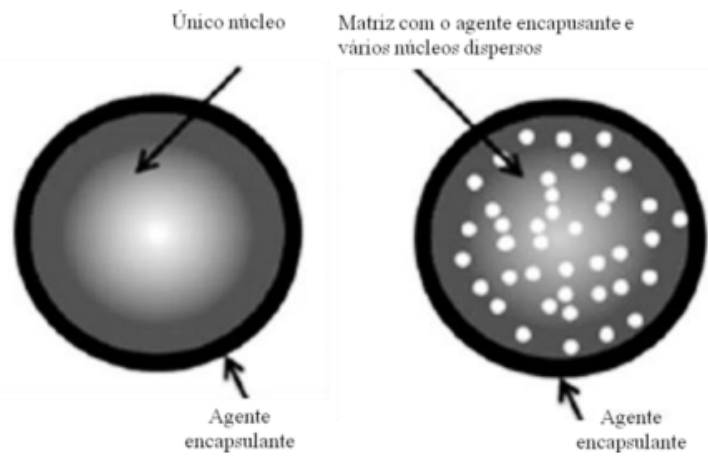

Figura 2 - Representação da estrutura das microcápsulas (a) mononucleares e (b) multinucleares. Fonte: Burgain et al. (2011)

Dessa forma, os métodos de microencapsulação devem ser empregados de acordo com a aplicação que será dada à micropartícula, o tamanho desejado, o mecanismo de liberação e as propriedades físico-químicas, tanto do material ativo quanto do agente encapsulante (FÁVARO-TRINDADE; PINHO; ROCHA, 2008).
O processo de microencapsulação por spray drying tem sido utilizado com sucesso na área de alimentos, tendo em vista a proteção de substâncias sensíveis à luz, oxigênio, temperatura e tempo de armazenamento, tais como os compostos fenólicos. Além disso, este processo pode impedir interações com outros compostos, promovendo uma maior estabilidade do produto e, consequentemente, aumentando a sua vida útil (DESAI; PARK, 2005). Estudos recentes têm mostrado que compostos bioativos, como os compostos fenólicos, podem ser protegidos por diferentes agentes encapsulantes no processo de microencapsulação por spray drying (SAÉNZ et al., 2009; BAKOWSK-BARCZAC; KOLODZIEJCZYK, 2011; ÇAM; ICYER; ERDOGAN, 2013; MISHRA; MISHRA; MAHANTA, 2013; PANG; YUSOFF; GIMBUN, 2014).

\section{Agentes encapsulantes}

No processo de microencapsulação tem se utilizado uma diversidade de agentes encapsulantes que são responsáveis pelo revestimento dos compostos ativos, além de conferir forma à microcápsula (AZEREDO, 2005). Os agentes encapsulantes podem ser de origem natural, semissintética ou sintética, incluindo materiais poliméricos, hidrófilos, hidrófobos ou uma associação de ambos (ANSON, 2005). Os agentes encapsulantes têm por função proporcionar proteção durante o armazenamento prolongado, evitando que ocorram alterações químicas e sensoriais no material encapsulado (GOUIN, 2004; SOUZA et al., 2005). Dentre os agentes encapsulantes mais estudados (sozinhos ou em associações) pelo método de spray drying, destacam-se os biopolímeros, provenientes de várias fontes como as gomas naturais (goma arábica, alginatos, carragenas), amidos, gelatina, derivados do leite, como o leite desnatado reconstituído e maltodextrinas (GHARSALLAOUI et al., 2007).

A escolha do agente encapsulante deve ser baseada, sobretudo nas características físicas e químicas do composto que se pretende encapsular (porosidade, solubilidade), no tipo de aplicação (fármaco, aditivo alimentar, entre outros) e no método de microencapsulação selecionado (SUAVE et al., 2006). Além disso, algumas características são necessárias para que o agente encapsulante seja considerado ideal, tais como apresentar baixa viscosidade em concentrações elevadas; ser fácil de manusear durante o processo de microencapsulação; apresentar baixa higroscopicidade para evitar aglomerações; ter elevada capacidade de incorporar o material a encapsular; proteger o material que será encapsulado de circunstâncias adversas, como oxigênio, luz e pH; não deve reagir com o composto a encapsular; apresentar propriedades desejadas de liberação do material a encapsular, ter sabor agradável quando administrado por via oral; não possuir aroma (SANTOS et al., 2000). Os agentes encapsulantes ainda devem possuir a capacidade de formar película coesa com o material do núcleo, proporcionando com- 
patibilidade química e física que vai conferir algumas propriedades desejadas às microcápsulas, tais como flexibilidade, resistência, impermeabilidade e estabilidade na preparação (VENKATESEN et al., 2009).

Estudos relataram a encapsulação do extrato de erva mate por diferentes métodos, utilizando distintos agentes encapsulantes, tais como sacarose, soluções de alginato de sódio e quitosana, soluções de alginato de sódio e amido de milho e alginato de cálcio (LOPEZ-CORDOBA; DELADINO; MARTINO, 2013; LÓPEZ-CORDOBA et al., 2014; DELADINO; NAVARRO; MARTINO, 2013; LÓPEZ-CORDOBA; DELADINO; MARTINO, 2014). No entanto, pelo método de spray drying, a utilização de maltodextrina vem se destacando por mostrar maior eficiência na proteção de compostos fenólicos (BAKOWSK -BARCZAC; KOLODZIEJCZYK, 2011; WU et al., 2014; PANG; YUSOFF; GIMBUM, 2014).

\section{Conclusões}

Ilex paraguariensis têm grande potencial antioxidante especialmente por apresentar altos teores de compostos fenólicos. Por este fato, estudos vêm demonstrando diversos benefícios associados à utilização desta planta. Assim, torna-se importante melhorar sua composição através da aplicação de tecnologias de concentração, no entanto, o aumento no conteúdo de compostos fenólicos presentes no extrato aquoso de erva mate resulta em maior instabilidade frente alguns fatores, tais como, presença de luz, oxigênio e temperatura, resultando em uma consequente degradação. Neste contexto, a tecnologia de microencapsulação por spray drying aparece como alternativa de grande potencial para exercer efeito protetor sobre estes compostos, e, além disso, microcápsulas contendo extrato aquoso crioconcentrado das folhas de erva mate, pode vir a representar um aditivo alimentar promissor para a incorporação em alimentos.

\section{Referências}

AIDER, M.;DEHALLEUX, D. Cryoconcentration technology in the bio-food industry: principles and applications. LWTFood Science and Technology, v. 42, n. 3, p. 679-685, 2009.

AIDER, M.; DE HAlleUX, D. Production of concentrated cherry and apricot juices by cryoconcentration technology. LWT - Food Science and Technology, v. 41, n. 10, p. 1768-1775, 2008.

AIDER, M.; DE HALLEUX, D.; AKBACHE, A. Whey cryoconcentration and impact on its composition. Journal of Food Engineering, v. 82, n. 1, p. 92-102, 2007.

AIDER, M.; DE HALLEUX, D.; MELNIKOVA, I. Skim milk whey cryoconcentration and impact on the composition of the concentrated and ice fractions. Food and Bioprocess Technology, v. 2, n. 1, p. 80-88, 2009.

ANANDARAMAN, S.; REINECCIUS, G.A. Stability of encapsulated orange peel oil. Food Technology, v.40, p.88-93, 1986.

ALEX, L. C.; LORENA, D.; MIRIAN, M. Effect of starch filler on calcium-alginate hydrogels loaded with yerba mate antioxidants, Carbohydrate Polymers, v. 95, n. 1, p. 315-323, 2013.

ANESINI, C.; TURNER, S.; COGOI, L.; FILIP, R. Study of the participation of caffeine and polyphenols on the overall antioxidant activity of mate (Ilex paraguariensis). Food Science and Technology, v. 2, n. 45, p. 299 - 304, 2012.

ANON, A. Freeze concentration opens new product opportunities. Journal of Food Engineering, v.65, n.3, p.50-52, 1993.

ANSON, R. Microencapsulation: for enhaced textile performance. Performance Apparel Markets, v. 12, n, 12, p. 21-39, 2005.

ARCARI, D. P. et al. Antiobesity effects of yerba mate extract (Ilex paraguariensis) in high-fat diet-induced obese mice. Obesity (Silver Spring), v. 17, n.12, p. 2127-2133, 2009.

AZEREDO, H. M. C. Encapsulação: aplicação à tecnologia de alimentos. Alimentos e Nutrição, v. 16, n. 1, p. 89-95, 2005.

BAKOWSKA, A. M.; KUCHARSKA, A. Z.; OSZMIANSKI, J. The effects of heating, UV irradiation and storage on stability of anthocyanin-polyphenol copigment complex. Food Chemistry, v. 81, n. 3, p. 349-355, 2003.

BAKOWSKA-BARCZK; KOLODZIEJCZYK. Black currant polyphenols: their storage stability and microencapsulation. Industrial Crops and Products, 34, 3101-3109, 2011.

BANSODE, S. S.; BANARJEE, S. K.; GAIKWAD, D. D.; JADHAV, S. L.; THORAT, R. M. Microencapsulation: a review. International Journal of Pharmaceutical Sciences Review and Research, v. 1, n. 2, p. 38-43, 2010.

BASTOS, D. H, SALDANHA, L. A.; CATHARINO, R. R; SAWAYA, A.; CUNHA, I. B.; CARVALHO, P. O.; EBERLIN, M. N. Phenolic antioxidants identified by ESI-MS from Yerba Maté (Ilex paraguariensis) and green tea (Camellia sinensis) extracts. Molecules, v. 3, n. 12, p. 423 - 432, 2007.

BELÉN, F.; SÁNCHEZ, J.; HERNÁNDEZ, E.; AULEDA, J. M.; RAVENTÓS, M. One option for the management of wastewater from tofu production: Freeze concentration in a falling-film system. Journal of Food Engineering, v. 110, n. 3, p. 364-373, 2012. 
BENZIE, I. F. F.; STRAIN, J. J. The ferric reducing ability of plasma (FRAP) as a measure of antioxidant power: the frap assay. Analytical Biochemistry, v. 239, p. 70-76, 1996.

BERTÉ, K. A. S. 2011. Tecnologia da Erva Mate solúvel. 160f. Tese (Doutorado em Tecnologia de Alimentos) - Setor de Tecnologia, Universidade Federal do Paraná, 2011.

BOAVENTURA, B. C. et al. Enhancement of bioactive compounds content and antioxidant activity of aqueous extract of mate (Ilex paraguariensis A. St. Hil.) through freeze concentration technology. Food Research International, v. 23, n. 2, p. 686-692, 2013.

BOAVENTURA, B. C. et al. Association of mate tea (Ilex paraguariensis) intake and dietary intervention and effects on oxidative stress biomarkers of dyslipidemic subjects. Nutrition, v. 28, n. 6, p. 657-664, 2012.

BRACESCO, N. et al. Antioxidant activity of a botanical extract preparation of Ilex paraguariensis, prevention of DNA double-strand breaksin Saccharomyces Cerevisiae and human low-density lipoprotein oxidation. Journal of Alternative and Complementary Medicine. v. 9, p. 378-387, 2003.

BRACESCO, N.; SANCHEZ, A. G.; CONTRERAS, V.; MENINI, T.; GUGLIUCCI, A. Recent advances on Ilex paraguariensis research: Minireview. Journal of Ethnopharmacology, v. 136, p. 378 - 384, 2011.

BRAND-WILLIAMS, W.; CUVELIER, M. E.; BERSET, C. Use of a free-radical method to evaluate antioxidant activity. LWT-Food Science and Technology, v. 28, n. 1, p. 25-30, 1995.

BRASIL. Ministério do Planejamento, Orçamento e Gestão. Produção da extração vegetal e Silvicultura. Instituto Brasileiro deGeografia e Estatística-IBGE, v. 26, p. 1-55, 2011.

BRASIL. Secretária da Agricultura e do Abastecimento - Governo do Estado do Paraná. Produtos Florestais - Erva-mate. Disponível em: http://www.agricultura. pr.gov.br/arquivos/File/deral/Prognosticos/Erva_ Mate_2013_2014.pdf. Acesso em: 10 de junho de 2014.

BRAVO, L.; GOYA, L.; LECUMBERRI, E. LC/MS characterization of phenolic constituents of mate (Ilex paraguariensis St. Hil.) and its antioxidant activity compared to commonly consumed beverages. Food Research International, v. 40, n. 3, p. 393-405, 2007.

BURGAIN, J.; GAIANI, C.; LINDER, M.; SCHER, J. Encapsulation of probiotic living cells: from laboratory scale to industrial applications. Journal of Food Engineering, v. 104, p. 467-483, 2011.

ÇAM, M.; IÇYER, N. IÇYER, N. C.; ERDOGAN, F.
Pomegranate peel phenolics: microencapsulation, storage stability and potential ingredient for functional food development. LWT - Food Science and Technology, v. 55, p. 117-123, 2013.

CAMPOS, R. M. L. et al. Fatty acid and volatile compounds from salami manufactured with yerba mate (Ilex paraguariensis) extract and pork back fat and meat from pigs fed on diets with partial replacement of maize with rice bran. Food Chemistry, v. 103, p.1159-1167, 2007.

CARARETO, N. D. D.; MONTEIRO FILHO, E. S.; PESSÔA FILHO, P. A.; MEIRELLES, A. J. A. Water actitivity of aqueous solutions of ethylene oxide-propylene oxide block copolymers and maltodextrins. Brazilian Journal of Chemical Engineering, v. 27, p. 173-181, 2010.

CARVALHO, P. E. R. Espécies Arbóreas Brasileiras. Brasília: Emprapa Informação Tecnológica; Colombo, PR: Embrapa Florestas, v. 1, 2003. 1039 p.

CHABAROV, A.; AIDER, M. Mathematical modeling and experimental validation of the mass transfer during unidirectional progressive cryoconcentration of skim milk. Innovative Food Science \& Emerging Technologies, v. 21, p. 151-159, 2014.

CLIFFORD, M. N. Diet-derived phenols in plasma and tissues and their implications for health. Planta Medica, v. 79, p.1103-1114, 2004.

CLIFFORD, M.N.; RAMIREZ-MARTINEZ, J.R. Chlorogenic acids and purine alkaloids contents of mate (Ilex paraguariensis) leaf and beverage. Food Chemistry, v. 35, p. 21, 1990.

CÓRDOBA-LÓPEZ, A.; DELADINO, L.; AGUDELOMESA, L.; MARTINO, M. Yerba mate antioxidant powders obtained by co-crystallization: Stability during storage. Journal of Food Engineering, v. 124, p. 158-165, 2014.

CORREA, G. et al. Carbona 4: Desenvolvimento de uma progênie biclonal de erva-mate em Machadinho, RS. Colombo, Paraná: Embrapa Florestas, 2011 (Série Documentos).

DA SILVA, E. L.; NEIVA, T. J.; SHIRAI, M.; TERAO, J.; ABDALLA, D. S. P. Acute ingestion of yerba mate infusion (Ilex paraguariensis) inhibits plasma and lipoprotein oxidation. Food Research International, v. 41, n. 10, p. 973-979, 2008.

DE MEJÍA, E. G.; SONG, Y. S.; HECK, C. I.; RAMIREZ-MARES, M. V. Yerba mate tea (Ilex paraguariensis): phenolics, antioxidant capacity and in vitro inhibition of colon cancer cell prolifetation. Journal of Functional Foods, v.1, n. 2, p. 23 - 24, 2009. 
DE MORAIS, E. C. et al. Consumption of yerba mate (Ilex paraguariensis) improves serum lipid parameters in healthy dyslipidemic subjects and provides an additional LDL-cholesterol reduction in individuals on statin therapy. Journal of Agricultural and Food Chemistry, v. 57, n. 18, p. 8316-8324, 2009.

DELADINO, L.; NAVARRO, A. S.; MARTINO, M. N. Carrier systems for yerba mate extract (Ilex paraguariensis) to enrich instant soups. Release mechanisms under different $\mathrm{pH}$ conditions. LWT - Food Science and Technology, v. 53, n. 1, p. 163-169, 2013.

DERMARDEROSIAN, A. The Review of Natural products. St Louis: Facts \& Comparisons, p. 396-397, 2001.

DESAI, K. G.H.; PARKA, H. J.Recent developments in microencapsulation of food ingredients. Drying Technology, v. 23, n. 7, p. 1361-1394, 2005.

DUDONNÉ, S.; VITRAC, X.; COUTIERÉ, P.; WOILLEZ, M.; MÉRILLON, J. M. Comparative study of antioxidant properties and total phenolic content of 30 plant extracts of industrial interest using DPPH, ABTS, FRAP, SOD and ORAC assays. Journal of Agricultural and Food Chemistry, v.57, p.1768-1774, 2009.

ELNAGGAR, Y.S.R. EL-MASSIK, A.M. ABDALLAH O.Y. EBIAN, A.E.R. Maltodextrin: a novel excipient used in sugarbased orally disintegrating tablets and phase transition process. AAPSPharmSciTech, v.11 p. 645-651,2010.

ERSUS, S.; YURDAGEL, U. Microencpasulation of anthocyanin pigments of black carrot (Daucuscarota L.) by spray dryer. Journal food Engineering, n. 80, p. 805-812, 2007.

FANG, Y. Z.; YANG, S.; WU, G. Free radicals, antioxidants, and nutrition. Nutrition, v. 18, p. 872-879, 2002.

FANG, Z.; BHANDARI, B. Encapsulation of polyphenols: a review. Trends in Food Science \& Technology, v. 21, n. 10, p, 510-523, 2010.

FÁVARO-TRINDADE, C. S.; PINHO, S. C.; ROCHA, G. A. Revisão: Microencapsulação de ingredientes alimentícios. Brazilian Journal of Food Technology, v. 11, p. 103-112, 2008.

FERNANDES, E.S.; DE OLIVEIRA MACHADO, M.; BECKER, A. M.; DE ANDRADE, F.; MARASCHIN, M.; DA SILCA, E. L. Yerba mate (Ilex paraguariensis) enhances the gene modulation and activity of paraoxonase-2: In vitro and in vivo studies. Nutrition, v. 28, p. 1157-1164, 2012.

FERREIRA, F.; VÁZQUEZ, V.; GUNTNER, C.; MOYNA, P. Inhibition of the passive diffusion of cholic acid by the Ilex paraguariensis St. Hil. saponins. Phytotherapy Research, v. 11, n. 2, p. 79-81, 1997.
FILIP, R. 1.; LOTITO, S. B.; FERRARO, G.; FRAGA, C. G. Antioxidant activity of Ilex paraguariensis and related species. Nutrition Research, v. 20, n. 10, p. 1437-1446, 2000.

FILIP, R.; DAVICINO, R.; ANESINI, C. Antifungal activity of the aqueous extract of Ilex paraguariensis against Malassezia furfur. Phytotherapy Research,v. 24, p.715-719, 2010.

FRANCIS, G.; KEREM, Z.; MAKKAR, H. P. S.; BECKER, K. The biological action of saponins in animal systems: a review. British Journal of Nutrition, v. 88, n. 6, p. 587-605, 2002.

FRYER, P. J.; ROBBINS, P. T. Heat transfer in food processing: ensuring product quality and safety. Applied Thermal Engineering, v. 25, p. 2499-2510, 2005.

GHARSALLAOUI, A.; ROUDAUT, G.; CHAMBIN, O.; VOILLEY, A.; SAUREL, R. Applications of spray-drying in microencapsulation of food ingredients: an overview. Food Research International, v.40, p. 1107-1121, 2007.

GNOATTO, S. C. B. et al. Evaluation of ursolic acid isolated from Ilex paraguariensis and derivatives on aromatase inhibition. Europan Journal of Medicinal Chemistry, 2008.

GUCLU-USTUNDAG, O.; MAZZA, G. Saponins: properties, applications and processing. Critical Reviews in Food Science and Nutrition, v. 47, n. 3, 2007.

GUGLIUCCI, A.; STAHL, A. J. C. Low-densitylipoprotein oxidation is inhibited by extracts of Ilex paraguariensis. Biochemistry and Molecular Biology International, v. 35, n. 1, p. 47-56, 1995.

HECK, C.I., de MEJIA, E.G. Yerba Mate Tea (Ilex paraguariensis), a comprehensive review on chemistry, health implications, and technological considerations. Journal of Food Science, v.72, p.138-151, 2007.

HECK, C.I.; SCHMALKO, M.; GONZALEZ DE MEJIA, E. Effect of growing and drying conditions on the phenolic composition of mate teas (Ilex paraguariensis). Journal of Agricultural and Food Chemistry, V. 56, P. 8394-8403, 2008.

HIMAWAN, C. et al. Dynamic modeling and simulation of eutectic freeze crystallization. Journal of Crystal Growth, v. 237-239, n. 1-4 III, p. 2257-2263, 2002.

HUANG, D.; OU, B.; PRIOR, R. L. The Chemistry behind antioxidant capacity assays. Journal of Agricultural and Food Chemistry, v.53, n.6, p.1841-1856, 2005.

KANG, Y. R. et al. Antiobesity and anti-diabetic effects of yerba mate (Ilex paraguariensis) in C57BL/6J mice fed a high-fat diet. Laboratory Animal Research, v. 28, n. 1, p. 23-29, 2012. 
KILMARTIN, P.A. REID, D.S. SAMSON, I. Dielectric properties of frozen maltodextrin solutions with added $\mathrm{NaCl}$ across the glass transition. Journal of the Science of Food and Agriculture, v.84, p. 1277-1284, 2004.

KISSEL, T.; MARETSCHEK, S.; PACKHAUSER, C.; SCHNIEDERS, J.; SEIDEL, N. Microencapsulation techniques for parenteral depot systems and their application in the pharmaceutical industry. In: BENITA, S. Microencapsulation: Methods and Industrial Applications. $2^{\circ}$ edição. Boca Raton, CRC Press Taylor E Francis Group, pp. 104, 2006.

KUANG, S. S.: OLIVEIRA, J. C.; CREAN, A. M. Microencapsulation as a tool for incorporating bioactive ingredients into food. Critical Reviews in Food Science and Nutrition, v. 50, n. 10, p. 1913-1918, 2010.

LEOPOLDINI, M.; RUSSO, N.; TOSCANO, M. The molecular basis of natural polyphenolic antioxidants. Food Chemistry, v. 125, p. 288-306, 2011.

LEWICKI, P. P. Design of hot air drying for better foods. Trends in Food Science \& Technology, v. 17, p.153-163, 2006.

LOPÉZ-CORDOBA, A.; DELADINO, L.; AGUDELOMESA, L.; MARTINO, M. Yerba mate antioxidant powders obtained by co-crystallization: Stability during storage. Jounal of Food Engineering, v. 124, p. 158-165, 2014.

LÓPEZ-CORDOBA, A.; DELADINO, L.; MARTINO, M. Effect of starch filler on calcium-alginate hydrogels loaded with yerba mate antioxidants. Carbohydrate Polymers, v. 95, n. 5, p. 315-323, 2013.

LÓPEZ-CORDOBA, A.; DELADINO, L.; MARTINO, $M$. Release of yerba mate antioxidants from com starch-alginate capsules as affected by structure. Carbohydrate Polymers, v. 99, n. 2, p. 150-157, 2014.

MADENE, A.; MURIEL, J.; SCHER, J.; DESOBRY, $S$. Flavour encapsulation and controlled release - a review. International Journal of Food Science and Technology, v. 41, n. 1, p. 1-21, 2006.

MATSUMOTO, R. L. et al. Effects of mate tea (Ilex paraguariensis) ingestion on mRNA expression of antioxidant enzymes, lipid peroxidation, and total antioxidants status in healthy young women. Journal of Agricultural and Food Chemistry, v. 57, p. 1775-1780, 2009.

MEINHART, A. D. et al. Methylxanthines and phenolics content extracted during the consumption of mate (IIex paraguariensis A. St.HiI) beverages. Journal of Agricultural and Food Chemistry, v. 58, n. 4, p. 2188-2193, 2010.

MEJIA, E. G.; SONG, Y. S.; HECK, C. I.;
RAMIREZ-MARES, M. V. Yerba mate tea (Ilex paraguariensis): phenolics, antioxidant capacity and in vitro inhibition of colon cancer cell proliferation. Journal of Functional Foods, v. 2, p. 23-34, 2010.

MENINI, T.; HECK, C.; SCHULZE, J.; DE MEJIA, E.; GUGLIUCCI, A. Protective action of Ilex paraguariensis extract against free radical inactivation of paraoxonase- 1 in high-density lipoprotein. Planta Medica, v. 73, n. 11, 2007.

MIRANDA, D. D. et al. Protective effects of mate tea (Ilex paraguariensis) on $\mathrm{H} 2 \mathrm{O} 2$-induced DNA damage and DNA repair in mice. Mutagenesis, v. 23, n. 4, p, 261-265, 2008.

MISHRA, P.; MISHRA, S.; MAHANTA, C. L. Effect of maltodextrin concentration and inlet temperature during spray drying on physicochemical and antioxidant properties of amla (Emblica officinalis) juice powder. Food and Bioproducts Processing, v. 92, p. 252-258, 2013.

MIYAWAKI, O.; LIU, L.; SHIRAI, Y.; SAKASHITA, S.; KAGITANI, K. Tubular ice system for scaleup of progressive freeze-concentration. Journal of Food Engineering, v. 69, p. 107-113, 2005.

MOLYNEUX, P. The use of the stable free radical diphenylpicrylhydrazyl (DPPH) for estimating antioxidant activity. Songklanakarin Journal Science Technology, v. 26, n. 2, p. 211-219, 2004.

MORENO, F. L.; HERNÁNDEZ, E.; RAVENTÓS, M.; ROBLES, C.; RUIZ, Y. A process to concentrate coffe extract by the integration og falling film and block freeze-concentration. Journal of Food Engineering, v. 128, p. 88-95, 2014.

MORETTI, C. L.; ARAUjO, A. L.; MATTOS, L. M. Evolution of diferente oxygen. Carbon dioxide and nitrogen combinations employed to extend the shelf life of fresh-cut collard greens. Horticultura Brasileira, v. 21, n. 4, p. 676-680, 2003.

MOSIMANN, A. L.; WILHELM-FILHO, D.; DA SILVA, E. L. Aqueous extract of Ilex paraguariensis attenuates the progression of atherosclerosis in cholesterolfed rabbits. BioFactors, v. 26, n. 1, p. 59-70, 2006.

MUNIN, A.; EDWARDS-LÉVY, F. Encapsulation of natural polyphenolic compounds: a review. Pharmaceutics, v. 3, p. 793-829, 2011.

MURAKAMI, A. N. N. et al. Concentration of phenolic compounds in aqueous mate (Ilex paraguariensis A. St. Hil) extract through nanofiltration. LWT- Food Science and Technology, v. 44, n. 10, p. 2211-2216, 2011. 
thawing as a path to concentrate aqueous solution. Separation and Purification Technology, v. 73, n. 3, p. 403-408, 2010.

OBEIDAT, W. N. Recent patents review in microencapsulation of pharmaceuticals using de emulsion solvent removal methods. Recent Patents on Drug Delivery \& Formulation, v. 3, n. 3, p. 178-192, 2009.

OLTHOF, M. R. et al. Chlorogenic acid, quercetin-3-rutinoside and black tea phenols are extensively metabolized in humans. The Journal of Nutrition, v. 133, p. 1806-1814, 2001.

PAGANINI STEIN, F. L. et al. Vascular responses to extractable fractions of Ilex paraguariensis in rats fed standard and high-cholesterol diets. Biological Research Nursing, v. 7, n. 2, p. 146-156, 2005.

PANG, S. F.; YUSOFF, M. M.; GIMBUM, J. Assessment of phenolic compounds stability and retention during spray drying of Orthosiphon stamineus extracts. Food Hydrocolloids, v. 37, p. 159-165, 2014.

PEREIRA, D. F. R. et al. Influence of the traditional Brazilian drink Ilex paraguariensis tea on glucose homeostasis. Phytomedicine, v. 19, n. 10, 868-877, 2012.

PÉREZ-JIMÉNEZ， J.; SAURA-CALIXTO, F. Effect of solvent and certain food constituents on different antioxidant capacity assays. Food Research International, v. 39, n. 7, p. 791-800, 2006.

PIMENTEL, G. D. et al. Yerba mate extract (Ilex paraguariensis) attenuates both central and peripheral inflammatory effects of diet-induced obesity in rats. Journal of Nutritional Biochemistry, v. 24, n. 5, p. 809-818, 2012.

POMILIO, A. B.; TRAJTEMBERG, S.; VITALE, A. A. High-performance capillary electrophoresis analysis of mate infusions prepared from stems and leaves of Ilex paraguariensis using automated micellar electrokinetic capillary chromatography. Phytochemical Analysis, v. 13, n. 4, p. 235-241, 2002.

PRIOR, R. L.; WU, X.; SCHAICH, K. Standardized methods for the determination of antioxidant capacity and phenolics in foods and dietary supplements. Journal Agricultural Food Chemistry, v. 18, n. 45, p. 290-302, 2005.

PRUDÊNCIO, A. P. A. et al. Phenolic composition and antioxidant activity of the aqueous extract of bark from residues from mate tree (Ilex paraguariensis St. Hil.) bark harvesting concentrated by nanofiltration. Food and Bioproducts Processing, v. 90, n. 3, p. 399-405, 2012.

PUANGPRAPHANT, S. et al. Dicaffeoylquinic acids in yerba mate (Ilex paraguariensis St. Hill) inhibit
NF-kB nucleus translocation in macrophages and induce apoptosis by activating caspases- 8 and -3 in human colon cancer cells. Molecular Nutrition and Food Research, v. 55, n. 10, p. 1509-1522, 2011.

QI, Z.H. XU, A. Starch-based ingredients for flavor encapsulation. Cereal Foods World, v.44, p. 460-465, 1999.

RATES, S. M. K. Metilxantinas. In: SIMÕES, C. M. O.; SCHENKEL, E. P., et al (Eds.). Farmacognosia: da planta ao medicamento, Florianópolis: Editora da UFSC/Porto Alegre: Editora da UFRGS, 2007.

RÉ, M. I. Formulating drug delivery systems by spray drying. Drying Thecnology, v. 24, n. 4, p. 433-446, 2006.

RÉ, M.I. Microencapsulation by spray drying. Drying Technology, v.16, p.1195-1236, 1998.

RESENDE, M. D. V. et al. Programa de melhoramento da ervamate coordenado pela EMBRAPA resultados da avaliação genética de populações, progênies, indivíduos e clones. Colombo: Embrapa Florestas, circular técnica 43, 2000. 67 p.

ROBERT, P. et al. Encapsulation of polyphenols and anthocyanins from pomegranate (Punica granatum) by spray dryer. International Journal of Food Science and Technology, v. 45, p. 1386-1394, 2010.

ROGINSKY, V.; LISSI, E. A. Review of methods to determine chain breaking antioxidant activity in food. Food Chemisty, v.92, p.235-254, 2005.

ROTTA, E.; OLIVEIRA, Y. M. M. Cultivo da erva mate. Distribuição geográfica. Embrapa Florestas, ISSN 1678-8281, 2005. Disponível em: <http:// sistemasdeproducao.cnptia.embrapa.br/FontesHTML/ Ervamate/CultivodaErvaMate/02_distrib_ geografica.htm> acesso em: 30 de janeiro de 2013.

SAÉNZ, C.; TAPIA, S.; CHÁVEZ, J.; ROBERT, P. Microencapsulation by spray drying of bioactive compounds from cactus pear (Opuntia ficusindica). Food Chemistry, v. 114, p. 616-622, 2009.

SAN MARTÍN, R.; BRIONES, R. Industrial uses and sustainable supply of Quillaja Saponaria (Rosaceae) saponins. Economic Botanic, v. 53, n.3, p. 302-311, 1999.

SÁNCHEZ, J.; HERNÁNDEZ, E.; AULEDA, J.M.; RAVENTÓS, M. Freeze concentration of whey in a fallingfilm based pilot plant: process and characterization. Journal of Food Engineering, v.103, p.147-155, 2011.

SÁNCHEZ, J.; RUIZ, Y.; RAVENTÓS, M.; AULEDA, J. M.; HERNÁNDEZ, E. Progressive freeze concentration of 
orange juice in a pilot plant falling film. Innovative Food Science \& Emerging Technologies, v. 11, n. 4, p. 644-651, 2010.

SANTOS, D. T.; MEIRELES, M. A. A. Carotenoids pigments encapsulation: Fundamentals, techniques and recent trends. The Open Chemical Engineering Journal, v. 4, p. 42-50, 2010.

SCHENKEL, E. P.; GOSMAN, G.; ATHAYDE, M. L. Saponinas. In: SIMÕES, C. M. D. O.; SHENKEL, E. P.; GOSMANN, G.; MELLO, J. C. P. D.; MENTZ, L. A.; PETROVICK, P. R. (org.). Farmacognosia: da planta ao medicamento. Porto Alegre/Florianópolis: Editora da UFRGS/Editora da UFSC, 2003. p. 711-740.

SHAHIDI, F.; HAN, X. Q. Encapsulation of food ingredients. Critical Reviews in Food Science Nutrition, n. 33, p. 501-547, 1993.

SILVA F., J. M. Aperfeiçoamento tecnológico do processo de crioconcentração: modelagem e simulação. Campinas: UNICAMP, 2003. Tese (Doutoradoem Engenharia Química), Programa de Pós Graduação em Engenharia QuímicaFaculdade de Engenharia Química. UNICAMP, 2003.

SILVA, C.; RIBEIRO, A.; FERREIRA, D.; VEIGA, F. Administração oral de proteínas e peptídeos: II. Aplicação de métodos de microencapsulação. Brazilian Journal of Pharmaceutical Sciences, v. 39, n. 1, p. 1-9, 2003.

SILVA, R. D.; BUENO, A. L.; GALLON, C. W.; GOMES, L. F.; KAISER, S.; PAVEI, C.; ORTEGA, G. G.; KUCHARSKI, L. C.; JAHN, M. O. The effect of aqueous extract of gross and commercial yerba mate (Ilex paraguariensis) on intra-abdominal and epididymal fat and glucose levels in male wistar rats. Fitoterapia, v. 82, n. 6, p. 818-826, 2011.

SIMÕES, C. M. O. et al. Farmacognosia da Planta ao Medicamento. 2 ${ }^{\mathrm{a}}$ ed. Porto Alegre / Florianópolis: Editora Universidade / UFRGS / Editora da UFSC, pág. 433- 449/ 723-738, 2000.

SONAGLIO, D.; ORTEGA, G. G.; PETROVICK, P. R.; BASSANI, V. L. Desenvolvimento tecnológico e produção de fitoterápicos. In: SIMÕES, C. M. O.; SCHENKEL, E. P.; GOSMANN, G.; MELLO, J. C. P.; MENTZ, L. A.; PETROVICK, P. R. Farmacognosia - da Planta ao Medicamento. Porto Alegre: Editora da UFRG/Editora da UFSC, 2007. p. 289-326.

SOTO-VACA, A.; GUTIERREZ, A.; LOSSO, J.N.; XU, Z.; FINLEY, J.W. Evolution of phenolic compounds from color and flavor problems to health benefits. Journal of Agricultural and Food Chemistry, v. 60, p. 6658-6677, 2012.

SOUZA, T. C. R.; PARIZE, A. L.; BRIGHENTE, I. M. C.; FÁVERE, V. T.; LARANJEIRA, M. C. M. Chitosan microspheres containing the natural urucum pigment.
Journal of Microencapsulation, v. 22, p. 511-520, 2005. SPARG, S. G.; LIGHAT, M. E.; VAN STADEN, J. Biological activities and distribution of plant saponins. Journal of Ethnopharmacology, v. 94, p. 219-243, 2004.

SUAVE, J.; DALL'AGNOL, E. C.; PEZZIN, A. P. T.; SILVA, D. A. K.; MEIER, M. M, SOLD, V. Microencapsulação: inovação em diferentes áreas. Health and Environment Journal, v. 7, n. 2, p.12-20, 2006.

TIWARI, S.: GOEL, A.; JHA, K. K.; SHARMA, A. Microencapsulation techniques and its application: a review. The Pharma Research, v. 3, n. 12, 2010.

TRINDADE, C. S. F.; PINHO, S. C.; ROCHA, G. A. Review: microencapsulation of food ingredients. Brazilian Journal of Food Technology, v. 11, n. 2, p. 103-109, 2008.

TURAN, M.;ATES, A.; INANC, B. Fouling of reverse osmosis and nanofiltration membranes by dairy industru effluents. Water Science Technology, v, 45, n. 14, p. 355-360, 2002.

VALDUGA, A. T. Uso sustentado e processamento de Ilex paraguariensis St. Hil. São Paulo: Universidade Federal de São Carlos, 2002. Tese (Doutorado em Ciência), Programa de Pós Graduação em Ecologia e Recursos Naturais, Universidade Federal de São Carlos. São Paulo, 2002.

VENKATESAN, P.; MANAVALAN, R.; VALLIAPPAN, K. Microencapsulation: a vital tecnique in novel drug delivery system. Journal of Pharmaceutical Sciences and Research, v. 1, n. 4, p. 26-35, 2009.

VIEIRA, M. A.; ROVARIS, A. A.; MARASCHIN, M.; DE SIMAS, K. N.; PODESTÁ, R.; AMBONI, R. D.; BARRETO, O. L.; AMANTE, E. R. Chemical characterization of candy made of yerba mate (Ilex paraguariensis A. St. Hil.) residue. Journal of Agricultural and Food Chemistry, v. 56, p. 4637- 4642, 2008.

WANG, Y.J.; WANG, L. Structures and properties of commercial maltodrextrins from corn, potato, and rice starches. Starch-Starke, v.52, p.296-304, 2000.

WICHTL, M. Herbal Drugs and Phytopharmaceuticals: A handbook for practice on a scientific basis. 3. ed. Boca Raton: CRC, p. 366-368, 2003.

WILSON, N.; SHAH, N. P. Microencapsulation of vitamins. ASEAN Food Journal, v. 14, n. 1, p. 1-14, 2007.

WU, Y.;ZOU, L.; MAO, J.; LIU, S. Stability and encapsulation efficiency of sulforaphane microencapsulated by spray drying. Carbohydrate Polymers, v, 102, n. 15, p. 497-503, 2014.

XU, L.; WANG, S. The Ginkgo biloba extract concentrated by nanofiltration. Desalination, v. 184, p. 305-313, 2005. 